\title{
Radial probe EBUS versus CT-guided needle biopsy for evaluation of peripheral pulmonary lesions: an economic analysis
}

\author{
Daniel P. Steinfort*, , Danny Liew" and Louis B. Irving*
}

ABSTRACT: Selection of the optimal procedure for minimally invasive diagnosis of peripheral pulmonary lesions (PPLs) may be based on clinical factors; however, selection of diagnostic strategy may also be influenced by cost. Economic analysis of minimally invasive diagnosis of PPL has not been performed previously.

Decision-tree analysis was applied to compare downstream costs of endobronchial ultrasoundguided transbronchial lung biopsy (EBUS-TBLB) with computed tomography-guided percutaneous needle biopsy (CT-PNB). Calculations were based on real costs derived from patient data. Sensitivity analyses and probabilistic sensitivity analysis were undertaken to identify the more cost-beneficial approach for varying input parameter values. Cost-effectiveness calculations were based on estimated disutility, according to the wait-trade-off technique.

For base-case analysis, initial evaluation with CT-PNB was cost-beneficial (AU\$2,724 versus EBUS-TBLB AU\$2,748). The variable which exerted the most influence on cost-benefit outcomes was the cost of managing complications. CT-PNB remained the more cost-effective procedure at base-case parameters, although thresholds were identified during sensitivity analysis where EBUS-TBLB became more cost-effective.

The costs of EBUS-TBLB and CT-PNB to evaluate PPL appear to be equivalent, but specific clinicalradiologic factors known to influence procedural outcomes will influence cost-benefit outcomes. Further evaluation of patient preferences and their influence on cost-effectiveness are required.

KEYWORDS: Complications, cost analysis, endobronchial ultrasound, nonsmall cell lung cancer

eripheral pulmonary lesions (PPL) are focal radiographic opacities that may be characterised as nodules $(\leqslant 3 \mathrm{~cm})$ or masses $(>3 \mathrm{~cm})$. While referral for lobectomy in patients with a PPL with a very high pre-test probability of malignancy is suggested by some guidelines [1], resectional biopsy is not risk-free and may not be necessary in a significant number of patients with such lesions [2]. Screening studies using computed tomography (CT) show that up to $34 \%$ of such operations are performed for benign nodules [3-5].

Noninvasive tests, such as fluorodeoxyglucose positron emission tomography or dynamic CT with nodule enhancement, cannot distinguish benign disease from malignant disease with sufficient accuracy [2]. Consequently, attempts at minimally invasive diagnosis are strongly favoured. This may be achieved by either bronchoscopic or percutaneous approaches.
Percutaneous sampling is generally performed under CT-fluoroscopic guidance. Bronchoscopy may be aided by guidance methods such as fluoroscopy [6, 7], virtual bronchoscopy [8], endobronchial ultrasound (EBUS) [7], or electromagnetic navigation (EMN) [9]. The highest diagnostic yield is associated with EBUS and/or EMN guidance [9]. Availability of EMN remains very limited, partly owing to the significant expense associated with the technology and ongoing consumable costs.

The performance characteristics of EBUS bronchoscopy and CT-guided percutaneous needle biopsy (CT-PNB) have been well described, although only one study has previously compared the two modalities head-to-head [10]. This study concluded that the overall diagnostic accuracy of EBUS was non-inferior to CT-PNB, but that the complication rate following EBUS-guided

Earn CME accreditation by answering questions about this article. You will find these at the back of the printed copy of this issue or online at www.erj.ersjournals.com/site/misc/cmeinfo.xhtml

\section{AFFILIATIONS}

*Dept of Respiratory Medicine, Royal Melbourne Hospital, and

\#Dept of Medicine, Royal Melbourne Hospital/Western Hospital, University of Melbourne, Parkville, Australia.

CORRESPONDENCE

D.P. Steinfort

Dept of Respiratory Medicine, Level 1, Centre for Medical Research

Royal Melbourne Hospital

Parkville

Victoria 3050

Australia

E-mail: daniel.steinfort@mh.org.au

Received:

March 142012

Accepted after revision:

June 062012

First published online:

July 262012 
transbronchial lung biopsy (EBUS-TBLB) was significantly lower. In addition to clinical "performance", the optimal test for diagnosis of PPLs may also be influenced by the costs of individual procedures. Costs for EBUS-TBLB and CT-PNB have not been previously reported. In particular, the cost of managing complications, and the influence of this on procedural cost outcomes, is unknown. Such information is highly relevant to clinical decision making.

In this study, we undertook a cost-benefit and cost-effectiveness analysis of EBUS-TBLB for management of PPLs, compared to CT-PNB.

\section{METHODS}

\section{Study site}

The Royal Melbourne Hospital in Melbourne, Australia, is a tertiary referral centre for the diagnosis, staging and management of lung cancer, with substantial experience in both EBUS-TBLB and CT-PNB. The hospital serves a catchment area of over 600,000 people. Patients with suspected/known lung cancer are managed by a multidisciplinary team comprising respiratory physicians, thoracic radiologists, thoracic surgeons, medical oncologists and radiation oncologists. The multidisciplinary team manages approximately 300 patients with lung cancer per year.

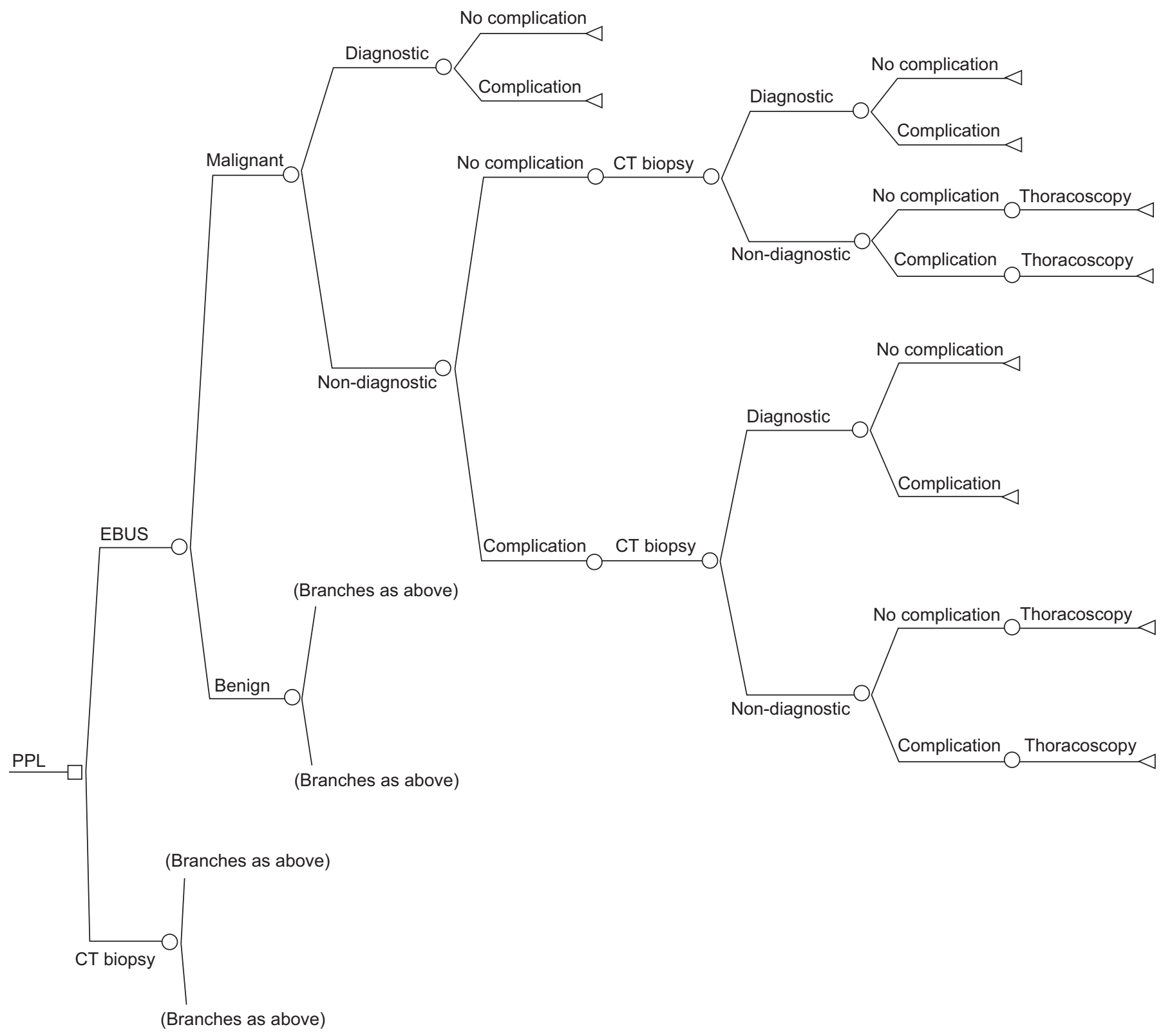

FIGURE 1. Decision tree illustrating possible clinical pathways following selection of either diagnostic approach. EBUS: endobronchial ultrasound; PPL; peripheral pulmonary lesion; CT: computed tomography. Square: decision node, i.e. the clinician may choose any clinical pathway for an individual patient. Circle: chance node, i.e. patients may experience either outcome, based on chance. The proportion of patients following each pathway from a chance node is dependent on pre-defined clinical parameters (table 3). Triangle: terminal node in the decision pathway, i.e. an individual patient has reached a definitive outcome in their diagnostic pathway. 


\begin{tabular}{lccc} 
TABLE 1 & \multicolumn{3}{l}{$\begin{array}{l}\text { Model input data: hospital costs associated with } \\
\text { uncomplicated procedures }\end{array}$} \\
\hline Procedure & Patients n & Median cost & $\begin{array}{c}\text { Updated cost } \\
\text { mean } \pm \text { SD }^{*}\end{array}$ \\
\hline $\begin{array}{l}\text { EBUS-TBLB } \\
\text { CT-PNB }\end{array}$ & 12 & 1318 & $1572 \pm 232$ \\
\hline
\end{tabular}

Data for median and updated cost are presented as AU\$. All procedures were completed as day admission cases. EBUS-TBLB: endobronchial ultrasoundguided transbronchial lung biopsy; CT-PNB: computed tomography-guided

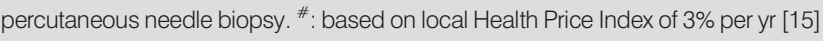

\section{Modelling approach}

Decision analysis, using specialised software (TreeAge Pro 2009, Excel module; TreeAge Software Inc., Williamstown, MA, USA), was applied to compare the downstream costs of EBUS-TBLB and CT-PNB (fig. 1) [11]. The pathway demonstrating lower healthcare costs (i.e. cost minimisation) is identified as the more cost-beneficial pathway. The analysis accounted for costs of each procedure, as well as costs incurred as a result of extra procedures required in the event of a negative result from either modality.

An advantage of decision tree analysis is its capacity to simulate even complex clinical algorithms, such as that for the evaluation of PPL. Furthermore, it can explicitly capture the uncertainty that is inherent in modelling of any type [12].

\section{Model population}

The modelled population comprised hypothetical patients referred to a multidisciplinary team for evaluation of PPL, for whom the team felt investigation was warranted and that either CT-PNB or EBUS would be acceptable modes of initial investigation of the lesion. Therefore, this excluded patients with the following features: 1) a clinical condition precluding investigation; 2) a lesion $<1 \mathrm{~cm}$ diameter anywhere in lung fields; 3) evidence on CT scan of central (endobronchially visible) lesion; and 4) other clinical site of disease more amenable to tissue diagnosis.

\section{Healthcare costs}

Unit cost estimates, in Australian dollars (AU\$), were based on recorded hospital costs for patients undergoing the abovementioned procedures at the Royal Melbourne Hospital between February 7, 2008 and January 22, 2010. All patients had provided written consent for inclusion in a randomised pragmatic trial comparing EBUS-TBLB with CT-PNB [10].

EBUS-TBLB and CT-PNB are performed on an outpatient basis at the Royal Melbourne Hospital. EBUS-TBLB is performed in a day procedure unit, with sedation administered by resident staff from the Respiratory Unit, as previously described [13]. The procedure itself has previously been described [14], using a 20-MHz radial EBUS probe (UM-BS20-26R; Olympus, Tokyo, Japan) and guide sheath. CT-PNB is performed using a coaxial needle (Bard TruGuide needle; Bard Biopsy Systems, Tempe, AZ, USA) and core biopsy instrument (Bard Biopy-Cut needle and Bard Magnum biopsy instrument; Bard Biopsy Systems).

Costs were derived from actual patient data at the Royal Melbourne Hospital, and includes both direct care costs (e.g. physician, nursing, radiology and pathology costs) as well as indirect costs such as equipment sterilisation and repair costs, and non-clinician staff costs (e.g. clerical or cleaning staff). Costing data for each patient admission was obtained from cost weight analysis compiled according to guidelines from the Clinical Costing Standards Association of Australia [15]. Hospital and median costs for EBUS-TBLB and CT-PNB were calculated based upon all patients included in a recently published randomised pragmatic trial [10]. Summary data for all uncomplicated procedures are shown in table 1. Costs for patients in whom complications occurred are shown in table 2. Costs for thoracoscopic resection were established following an audit of all patients undergoing thoracoscopy/thoracotomy for resection of lung lesions at the Royal Melbourne Hospital from July 1, 2007 to June 30, 2008. All costs were updated to 2010/ 2011 levels according to the locally recorded Health Price Index, which reported an increase of 3\% per year [16].

\section{Other input parameters}

Other input parameters applied to the decision tree analysis are described in table 3. Sensitivity and specificity of EBUS-TBLB for evaluation of PPL was based on our own experience and a published meta-analysis [10, 17], while data for CT-PNB was based on our reported experience and published guidelines [2, 10].

TABLE 2 Model input data: hospital costs associated with complicated procedures

\begin{tabular}{|c|c|c|c|c|}
\hline Procedure & Complication & Management & Length of stay days & Updated cost ${ }^{\#}$ \\
\hline EBUS-TBLB & Small, self-limiting pneumothorax & Conservative & 0 & 1941 \\
\hline CT-PNB & Small, self-limiting pneumothorax & Conservative & 0 & 1952 \\
\hline CT-PNB & Small, self-limiting pneumothorax & Conservative & 0 & 1791 \\
\hline CT-PNB & Hydropneumothorax & Conservative & 0 & 1905 \\
\hline CT-PNB & Haemothorax, pulmonary haemorrhage & Admission for analgesia and observation & 3 & 4932 \\
\hline
\end{tabular}


TABLE 3 Model input data: parameter values used for variables in performance of decision tree analysis

\begin{tabular}{|c|c|c|c|}
\hline Variable & Base-case value & Range utilised for sensitivity analysis & [Ref.] \\
\hline \multicolumn{4}{|l|}{ EBUS-TBLB sensitivity } \\
\hline Benign PPL & 0.50 & $0.50-0.80$ & \\
\hline \multicolumn{4}{|l|}{ CT-PNB sensitivity } \\
\hline Malignant PPL & 0.93 & $0.65-0.94$ & {$[2,10]$} \\
\hline Prevalence of malignancy & 0.87 & $0.5-0.95$ & {$[10,17]$} \\
\hline Mean cost of complications AU\$ & 327 & $300-3363$ & Current study \\
\hline
\end{tabular}

EBUS-TBLB: endobronchial ultrasound-guided transbronchial lung biopsy; PPL: peripheral pulmonary lesions; CT-PNB: computed tomography-guided percutaneous needle biopsy.

\section{Sensitivity analysis}

Calculations based on the above data constituted a "base-case" analysis, as defined by National Institute for Health and Clinical Excellence guidelines [18]. We recognised that model input values may vary significantly across different institutions. For example, diagnostic sensitivity of EBUS-TBLB differs considerably between institutions [17], and there is significant discrepancy in reported complication rates following CT-PNB [2]. Therefore, a series of one-way sensitivity analyses were undertaken within the range of each parameter recorded in table 3, based on data from recent pooled analyses. The values of these key inputs were varied one at a time, while maintaining the other inputs at "base-case" values. Subsequent analysis was undertaken to determine the threshold above which the most cost-beneficial approach remained in comparison to other diagnostic modalities.

Cost may also alter, depending on the severity of the condition, and institutional approaches to management (e.g. in- versus outpatient care and frequency of intercostal catheter insertion). Sensitivity analysis was performed to determine if a threshold cost for complications existed, above which the alternate investigation modality proved more cost-beneficial.
In order to assess the impact of uncertainty more accurately, probabilistic sensitivity analysis was performed using Monte Carlo simulations [19]. With this method, input parameters are assigned a distribution to reflect the nature of uncertainty. Multiple model simulations are then run. Monte Carlo simulation was performed using triangular distributions of values (lowest, likeliest, highest) as recorded in table 4 . With each simulation, one value from every input range is randomly sampled from within a specified data range according to its probability distribution. Thus, multiple outputs are generated, and uncertainty ranges are derived from the distributions of these. In our analysis, 10,000 simulations were undertaken.

\section{Cost-effectiveness}

The above methodology is used to assess the comparative costbenefit of competing diagnostic strategies for assessment of PPL. Cost-effectiveness requires consideration of quality-of-life measures. Patient preferences with regard to the impact of procedural complications or anxiety related to waiting for test results have been shown to influence cost-effectiveness analyses for patients with PPL [20].

\section{TABLE 4 Model input data: values used in Monte Carlo simulation}

\begin{tabular}{|c|c|c|c|c|}
\hline \multirow[t]{2}{*}{ Variable } & \multicolumn{3}{|c|}{ Values utilised in triangular probabilistic calculation } & \multirow[t]{2}{*}{ [Ref.] } \\
\hline & Lowest & Likeliest & Highest & \\
\hline Malignant PPL & 0.60 & 0.79 & 0.88 & {$[10,17]$} \\
\hline Benign PPL & 0.50 & 0.75 & 0.85 & \\
\hline \multicolumn{5}{|l|}{ CT-PNB sensitivity } \\
\hline Benign PPL & 0.56 & 0.80 & 0.90 & \\
\hline CT-PNB complication rate & 0.14 & 0.27 & 0.43 & {$[2,10]$} \\
\hline Mean cost of complications AU\$ & 300 & 654 & 3363 & Current study \\
\hline
\end{tabular}

EBUS-TBLB: endobronchial ultrasound-guided transbronchial lung biopsy; PPL: peripheral pulmonary lesions; CT-PNB: computed tomography-guided percutaneous needle biopsy. 
TABLE 5 Calculated costs of the two diagnostic approaches

\begin{tabular}{|c|c|c|c|c|c|c|c|c|c|c|}
\hline \multirow[t]{2}{*}{ Procedure } & \multirow{2}{*}{$\begin{array}{c}\text { Base-case } \\
\text { cost }\end{array}$} & \multirow[t]{2}{*}{ Range $^{\#}$} & \multicolumn{4}{|c|}{ Monte Carlo simulation results } & \multicolumn{4}{|c|}{ Patient scenario outcomes } \\
\hline & & & Mean $\pm S D$ & 10th centile & Median & 90th centile & Scenario 1 & Scenario 2 & Scenario 3 & Scenario 4 \\
\hline EBUS-TBLB AU\$ & 2748 & 2719-3534 & $2843 \pm 301$ & 2482 & 2814 & 3253 & 2482 & 2482 & 2814 & 2814 \\
\hline CT-PNB AU\$ & 2724 & 2683-3868 & $2935 \pm 340$ & 2515 & 2911 & 3385 & 2515 & 2515 & 2911 & 2911 \\
\hline
\end{tabular}

Cost-effectiveness outcomes are expressed in cost per qualityadjusted life-year (QALY), with utility being the measure on which quality adjustment is based. Utility allows adjustment of life-years gained by an intervention when those gained years would be lived in less than perfect health. Extra life-years are given a utility value of between 0 and 1 to account for this. This method is suitable for assessment of chronic health/disease states, although it is not able to assess the cost impact of shortterm disease states, such as pain or complications arising from a diagnostic procedure, or the anxiety resulting from a nondiagnostic procedure [21].

Multiple methods for assessment of the impact of transient disease states have been described. With the time-trade-off (TTO) technique, a patient decides between a longer period of time in less optimal health versus a shorter period in good health. A variation, the wait-trade-off technique, quantifies patients' preference for undergoing a particular test or treatment that has associated discomfort or restrictions that the patient may dislike. The patient is asked to trade-off extended time with the condition being diagnosed or treated in order to avoid the noxious effects of the test or treatment in favour of a similarly effective test or treatment but one not having side-effects [22]. A QALY toll is reflected in the

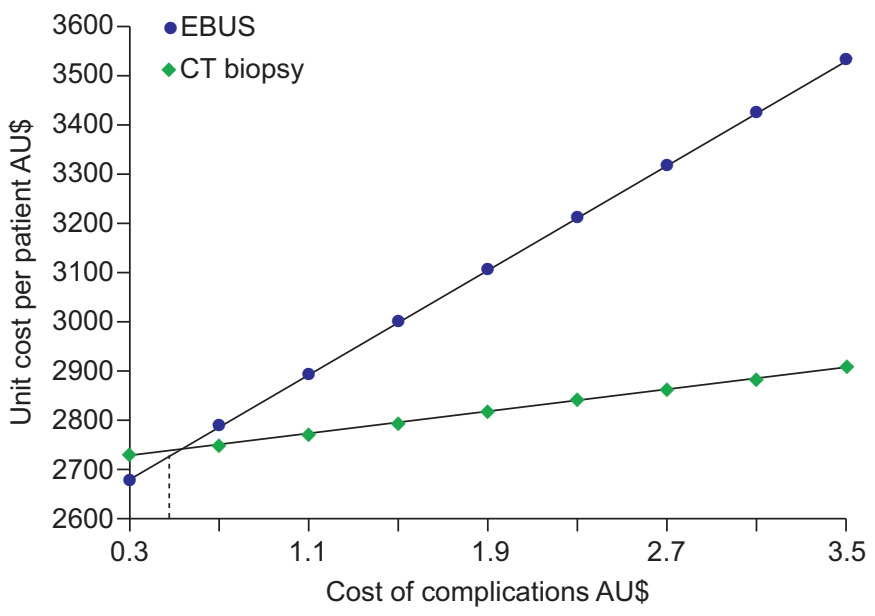

FIGURE 2. Effect on expected cost of each procedure during one-way sensitivity analysis (variation) in the cost of managing complications among the modelled population. Endobronchial ultrasound (EBUS)-guided transbronchial lung biopsy is cost-beneficial (i.e. cheaper) if mean cost of complications exceeds $\$ 501$ per episode. CT: computed tomography. wait-trade-off by an individual's willingness to wait longer to avoid more noxious experiences [23] and may be measured by disutility, being the fraction of a year of perfect health a patient would be willing to give up to avoid having to undergo a diagnostic test and to avoid its short-term morbidity [24]. This tool was originally designed for use in states related to diagnostic screening and testing [25]

Sensitivity analysis was performed for disutility, starting at a theoretical disutility of 0 for both the procedure itself (that is no utility penalty), as well as disutility attributable to complications arising from the procedure. One-way sensitivity analysis was performed to identify theoretical thresholds that may influence cost-effectiveness outcomes.

\section{Assumptions}

As sensitivity analysis is based on theoretical patients, we were required to make some specific assumptions regarding the theoretical model population. Key assumptions in the analysis were as follows. 1) There was a well-defined outcome in each arm of our decision model, i.e. pathologic diagnosis of PPL. 2) The long-term outcomes (measures of effectiveness) were equivalent in each model arm, i.e. treatment and outcomes of all patients was similar regardless of how the diagnosis was determined. As previously recognised [26], a cost-benefit analysis that assumes competing diagnostic strategies has equivalent outcomes and focuses, thereafter, only on cost outcomes is the most appropriate form of economic analysis to use in this setting. 3) Once a diagnosis has been made, the downstream costs of medical care were the same, regardless of how the diagnosis was achieved. 4) Thoracotomy/thoracoscopy had a diagnostic accuracy of $100 \%$ in the evaluation of PPL. 5) Pathology costs were identical regardless of the method of tissue acquisition.

\section{RESULTS}

\section{Base-case analysis}

Costs of each procedure based on base-case parameters are shown in table 5. For the base-case analysis, initial evaluation with CT-PNB was cost-beneficial in comparison to EBUS-TBLB by a margin of $\$ 24$ (CT-PNB $\$ 2,724$ versus EBUS-TBLB $\$ 2,748$ ).

\section{Sensitivity analysis}

One-way sensitivity analysis identified threshold values at which EBUS-TBLB became more cost-beneficial, which included the cost of managing complications exceeding \$501 per episode, a complication rate of CT-PNB exceeding $40 \%$ and sensitivity of CT-PNB for detection of malignancy falling 

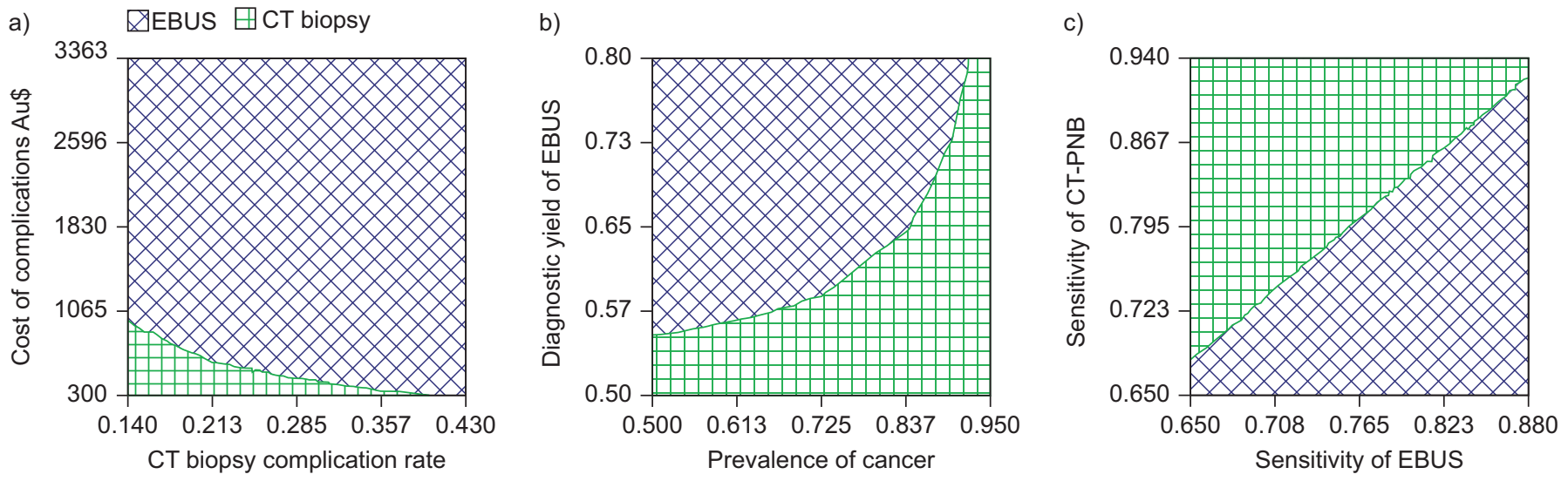

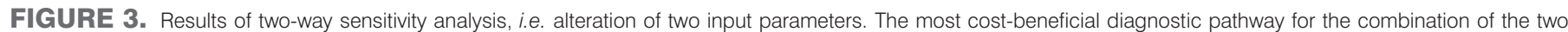

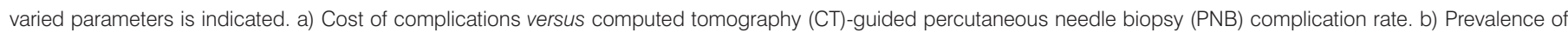

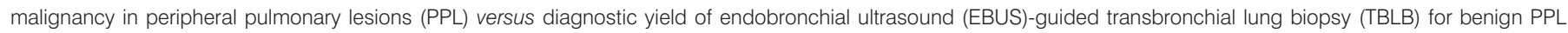
c) Sensitivity of EBUS-TBLB for detection of malignancy versus sensitivity of CT-PNB for detection of malignancy

below 91\%. Prevalence of malignancy had no effect on costbenefit during one-way analysis. Variation in diagnostic yield for benign disease had a negligible effect on outcomes for both procedures. The variable which exerted the most influence on cost outcomes was the cost of managing complications. The influence of this is shown in figure 2.

Two-way sensitivity analysis was undertaken to explore the interaction between two specific parameters. Threshold values are altered when two parameters are varied making identification of specific values impossible. The variation in cost-outcome with variation in both cost of complications, as well as complication rate of CT-PNB, is shown in figure 3a. Significant interaction was seen in two-way analysis with variation of prevalence of malignancy and sensitivity of EBUS-TBLB for detection of benign disease (fig. 3b), and with variation of sensitivity for detection of malignancy for both procedures (fig. 3c).

Given the influence of diagnostic sensitivity and complication rates on costs for procedures, we have modelled cost comparisons for hypothetical patient scenarios. The results are presented in table 5 . Input data for each scenario is presented in parentheses (EBUS-TBLB sensitivity, CT-PNB sensitivity and CT-PNB complication rate, respectively) and is based on published studies presented in table 6. Scenario 1: right lower lobe pleural-based nodule $(0.5,0.97$ and 0.03$)$. Scenario 2 : perihilar right middle lobe nodule $(0.88,0.85$ and 0.43$)$. Scenario 3: 6$\mathrm{cm}$ right upper lobe mass with "bronchus sign" $(0.9,0.8$ and $0.2)$. Scenario $4: 1.5$-cm proximal right lower lobe nodule, forced expiratory volume in $1 \mathrm{~s} 800 \mathrm{~mL}(0.7,0.7$ and 0.4$)$.

As expected, differing clinical scenarios resulted in different outcomes from cost comparisons. An increase in cost of managing complications above $\$ 327$, as used for these calculations, would result in increasing cost-benefit towards EBUS-TBLB due to the lower complication rate seen with this procedure.

\section{Probabilistic sensitivity analyses}

Outcomes of probabilistic sampling demonstrate the negligible difference in net costs between the two procedures (table 5). The two procedures differ by a maximum of $\$ 132$ when comparisons of mean, median and 10th and 90th centile values are made.

\section{Cost-effectiveness analysis}

Cost-effectiveness analysis was performed to examine the effect of disutility resulting from two potential adverse outcomes of the procedures: a non-diagnostic procedure (meaning further anxiety and the need for additional procedures), and a procedural complication (e.g. pneumothorax or hospital admission).

Using a theoretical wait-trade-off for a non-diagnostic procedure of 20 days (0.05 yrs), CT-PNB remained the more cost-effective procedure at base-case parameters. One-way sensitivity analysis in the range of values recorded in table 3 revealed that EBUSTBLB became the more cost-effective procedure if sensitivity of EBUS-TBLB for benign disease exceeded $71 \%$, if sensitivity of CT-PNB (malignancy) was below $89 \%$, or if cost of managing complications exceeded $\$ 560$. Unlike cost-benefit analyses, no threshold was observed for the complication rate of CT-PNB.

Using a theoretical wait-trade-off for a procedural complication of 20 days $(0.05 \mathrm{yrs}), \mathrm{CT}-\mathrm{PNB}$ remained the more costeffective approach $(\$ 2,778$ per QALY versus EBUS $\$ 2,816$ per QALY) at base-case parameters. One-way sensitivity analyses demonstrated that EBUS-TBLB became the more cost-effective approach if the cost of complications exceeded $\$ 489$, the complication rate for CT-PNB exceeded $40 \%$, and if the sensitivity of EBUS-TBLB for detection of benign disease exceeded $65 \%$. The effect in alteration of these two parameters (two-way sensitivity analysis) is demonstrated in figure 4.

As was demonstrated for cost-benefit calculations, the cost of managing complications was the input parameter that most heavily influenced the results of cost-effectiveness comparisons.

\section{DISCUSSION}

Our study was conducted in order to determine the most costbeneficial and cost-effective diagnostic procedure in the evaluation of PPL. Our analysis indicates that the two minimally invasive approaches used in evaluation of PPL differ in cost by negligible amounts, both in evaluation of the base-case scenario and following Monte Carlo probabilistic simulation.

The minimal differences between the two procedures observed in the base-case and probabilistic sensitivity analyses highlight the 


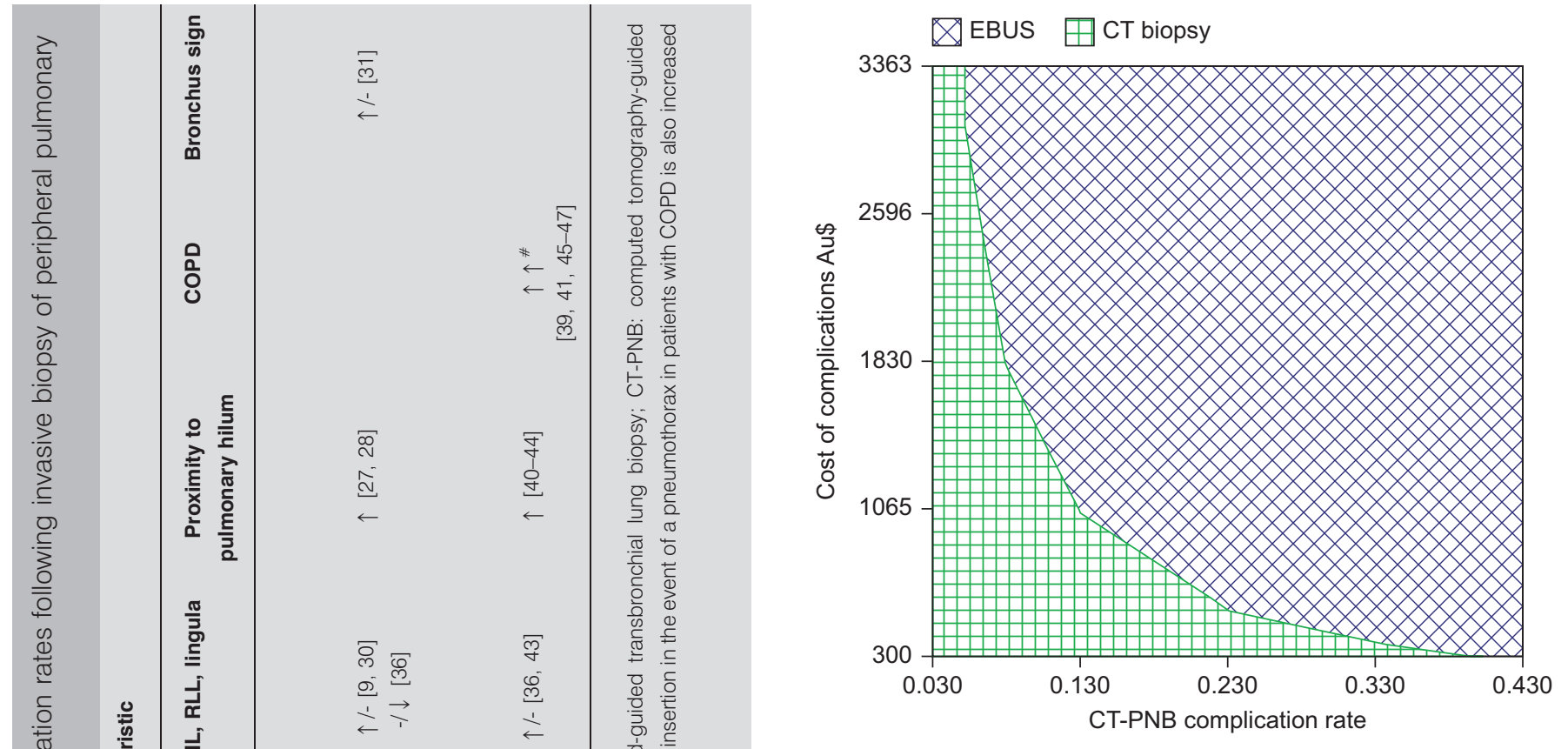

FIGURE 4. Disutility may be measured by the wait-trade-off technique. The most cost-effective procedure may then be determined on the basis of cost per quality-adjusted life-yrs. The graph illustrates the results of two-way sensitivity analysis, with cost-effectiveness measured according to disutility arising from procedural complications. A dynamic relationship is evident between the cost of complications and the complication rate of computed tomography-guided percutaneous needle biopsy (CT-PNB).

importance of clinical acumen in determining the most appropriate procedure. The only previously published randomised trial comparing EBUS-TBLB and CT-PNB found that overall diagnostic accuracy of EBUS-TBLB was non-inferior to CT-PNB [10]. However, numerous studies have demonstrated that both diagnostic accuracy and complication rates for both procedures may vary significantly, based on clinical factors (table 6).

At base-case values, CT-PNB enjoys an advantage by a having higher diagnostic sensitivity, while EBUS-TBLB has a lower complication rate. Specific clinical features are known to influence clinical outcomes and, therefore, will have an effect on cost outcomes. Clinical acumen may suggest to clinicians which procedure may serve a patient better (e.g. higher diagnostic sensitivity and lower risk of complications) and these factors will, as demonstrated in table 5, also predict favourable outcomes from a cost perspective.

Where cost and clinical outcomes may diverge is in assessment of cost-effectiveness. We have used theoretical values to conduct cost-effectiveness analysis using the wait-trade-off method. Modelling has previously indicated that cost-effectiveness of competing strategies depends on patient attitudes about taking risks [20]. To our knowledge, no published studies have examined the disutility value patients place on adverse outcomes, such as complications, or delay in diagnosis due to a non-diagnostic procedure.

Some patients may place a larger "cost" than 20 days (in the wait-trade-off methodology) on adverse outcomes, such that thresholds between the two methods may be significantly 
different to those recorded in our study. The "cost" of complications versus non-diagnostic procedures may differ considerably, and may be highly dependent on personality type. This also highlights the value of involving the patient in medical decision making, especially when clinical acumen suggests two approaches may be equivalent. Patients may prefer a procedure with higher diagnostic success even at the cost of a higher risk of complications, or more risk adverse patients may prefer a procedure with lower morbidity accepting a slightly higher likelihood of a non-diagnostic procedure.

Our analysis has demonstrated some factors that may influence the cost comparison between EBUS-TBLB and CTPNB. Cost of managing complications was the factor that most influenced cost-benefit results. A higher cost of complications favoured EBUS-TBLB in cost comparisons, due to the lower complication rate associated with this procedure. The cost of complications is likely to vary significantly between institutions, based on clinical practice (e.g. admission versus outpatient care) and cost of delivering care. Individual institutions and healthcare services may wish to undertake decision-tree analysis, based on local clinical and cost data, to determine their specific optimal investigative approach for patients with PPL.

\section{Strengths and limitations}

To our knowledge, this is the first cost comparison study of two minimally invasive procedures for evaluation of PPL. It is also the first to describe the cost of specific procedures, and costs associated with complications of these procedures.

Assumptions are required for decision tree analysis, and validity of the analyses is more certain when actual clinical data or variables are used instead of assumptions. Our analyses were well informed by our own local cost and clinical data, and sensitivity analysis allowed us to perform cost comparisons across most clinically realistic values, as described previously. We also accounted for the impact that false-negative results and procedural complications might have had.

Bronchoscopic staging of the mediastinum is cost-beneficial in comparison to the previous standard of surgical mediastinoscopy, largely as a minimally invasive approach to supplanting the significantly more expensive surgical procedure [26]. In contrast, we are comparing two minimally invasive procedures which are very similar in cost. Cost-benefit therefore relies on minimising "downstream" costs and, as illustrated in table 5, we have emphasised that clinical-radiologic factors known to influence procedural outcomes also strongly influence cost outcomes. Decision-tree analysis incorporating such information may assist clinical decision making, although this requires future study.

Our decision analysis model may aid clinicians in guiding local practice, but outcomes may vary considerably between institutions. Availability of local services, or expertise, may be a more pressing issue in determining clinical practice than our findings. Furthermore, individual patient characteristics may determine which specific modalities are most appropriate, regardless of cost concerns. Finally, patient preference will also guide clinical decision making. We attempted to account for the influence of patient preferences using measures of disutility to obtain cost-effectiveness values, but disutility has not been examined previously and should be included in future studies.

\section{Conclusions}

The costs of EBUS-TBLB and CT-PNB to evaluate PPL appear to be equivalent, but specific clinic-radiologic factors known to influence procedural outcomes will influence cost comparisons. Use of disutility scores to obtain QALY values did not significantly alter the outcome of cost-comparisons. Costminimisation relies on minimising "downstream" care costs. As a result, clinical acumen and incorporation of published data regarding influence of clinical-radiologic factors on procedural outcomes are likely to identify the most cost-beneficial diagnostic strategy. Further evaluation of patient preferences and their influence on cost-effectiveness are required.

\section{SUPPORT STATEMENT}

D.P. Steinfort was supported by a Postgraduate research scholarship from the National Health and Medical Research Council of Australia.

\section{STATEMENT OF INTEREST}

None declared.

\section{REFERENCES}

1 Gould MK, Fletcher J, Iannettoni MD, et al. Evaluation of patients with pulmonary nodules: when is it lung cancer? ACCP evidencebased clinical practice guidelines (2nd edition). Chest 2007; 132: Suppl. 3, 108S-130S.

2 Wahidi MM, Govert JA, Goudar RK, et al. Evidence for the treatment of patients with pulmonary nodules: when is it lung cancer? ACCP evidence-based clinical practice guidelines (2nd edition). Chest 2007; 132: Suppl. 3, 94S-107S.

3 Wilson DO, Weissfeld JL, Fuhrman CR, et al. The Pittsburgh Lung Screening Study (PLuSS): outcomes within 3 years of a first computed tomography scan. Am J Respir Crit Care Med 2008; 178: 956-961.

4 Swensen SJ, Jett JR, Hartman TE, et al. CT screening for lung cancer: five-year prospective experience. Radiology 2005; 235: 259-265.

5 Pastorino U, Bellomi M, Landoni C, et al. Early lung-cancer detection with spiral $\mathrm{CT}$ and positron emission tomography in heavy smokers: 2-year results. Lancet 2003; 362: 593-597.

6 Roth K, Hardie JA, Andreassen AH, et al. Predictors of diagnostic yield in bronchoscopy: a retrospective cohort study comparing different combinations of sampling techniques. BMC Pulm Med 2008; 8: 2.

7 Paone G, Nicastri E, Lucantoni G, et al. Endobronchial ultrasounddriven biopsy in the diagnosis of peripheral lung lesions. Chest 2005; 128: 3551-3557.

8 Asano F, Matsuno Y, Tsuzuku A, et al. Diagnosis of peripheral pulmonary lesions using a bronchoscope insertion guidance system combined with endobronchial ultrasonography with a guide sheath. Lung Cancer 2008; 60: 366-373.

9 Eberhardt R, Anantham D, Ernst A, et al. Multimodality bronchoscopic diagnosis of peripheral lung lesions: a randomized controlled trial. Am J Respir Crit Care Med 2007; 176: 36-41.

10 Steinfort DP, Vincent J, Heinze S, et al. Comparative effectiveness of radial probe endobronchial ultrasound versus CT-guided needle biopsy for evaluation of peripheral pulmonary lesions: a randomized pragmatic trial. Respir Med 2011; 105: 1704-1711.

11 Lilford RJ, Pauker SG, Braunholtz DA, et al. Decision analysis and the implementation of research findings. BMJ 1998; 317: 405-409.

12 Gouyer V, Conti M, Devos $\mathrm{P}$, et al. Tissue inhibitor of metalloproteinase 1 is an independent predictor of prognosis in 
patients with nonsmall cell lung carcinoma who undergo resection with curative intent. Cancer 2005; 103: 1676-1684.

13 Steinfort DP, Irving LB. Patient satisfaction during endobronchial ultrasound-guided transbronchial needle aspiration performed under conscious sedation. Respir Care, 55: 702-706.

14 Kurimoto N, Miyazawa T, Okimasa S, et al. Endobronchial ultrasonography using a guide sheath increases the ability to diagnose peripheral pulmonary lesions endoscopically. Chest 2004; 126: 959-965.

15 The Clinical Costing Standards Association of Australia. Clinical Costing Standards. www.ccsaa.com/standards.htm Date last accessed: February 15, 2012.

16 AIHW 2009. Health expenditure Australia 2007-08. Health and welfare expenditure series no. 37. Cat. no. HWE 46. Canberra, AIHW.

17 Steinfort DP, Khor YH, Manser RL, et al. Radial probe endobronchial ultrasound for the diagnosis of peripheral lung cancer: systematic review and meta-analysis. Eur Respir J 2011; 37: 902-910.

18 Andronis L, Barton P, Bryan S. Sensitivity analysis in economic evaluation: an audit of NICE current practice and a review of its use and value in decision-making. Health Technol Assess 2009: 13: 1-61.

19 Briggs AH. Handling uncertainty in cost-effectiveness models. Pharmacoeconomics 2000; 17: 479-500.

20 Raab SS, Hornberger J. The effect of a patient's risk-taking attitude on the cost effectiveness of testing strategies in the evaluation of pulmonary lesions. Chest 1997; 111: 1583-1590.

21 Cullen J, Schwartz MD, Lawrence WF, et al. Short-term impact of cancer prevention and screening activities on quality of life. J Clin Oncol 2004; 22: 943-952.

22 Swan JS, Fryback DG, Lawrence WF, et al. A time-tradeoff method for cost-effectiveness models applied to radiology. Med Decis Making 2000; 20: 79-88.

23 Swan JS, Sainfort F, Lawrence WF, et al. Process utility for imaging in cerebrovascular disease. Acad Radiol 2003; 10: 266-274.

24 Hrung JM, Langlotz CP, Orel SG, et al. Cost-effectiveness of MR imaging and core-needle biopsy in the preoperative work-up of suspicious breast lesions. Radiology 1999; 213: 39-49.

25 Wright DR, Wittenberg E, Swan JS, et al. Methods for measuring temporary health States for cost-utility analyses. Pharmacoeconomics 2009; 27: 713-723.

26 Steinfort DP, Liew D, Conron M, et al. Cost-benefit of minimally invasive staging of non-small cell lung cancer: a decision tree sensitivity analysis. J Thorac Oncol, 5: 1564-1570.

27 Fielding DI, Robinson PJ, Kurimoto N. Biopsy site selection for endobronchial ultrasound guide-sheath transbronchial biopsy of peripheral lung lesions. Internal Med J 2008; 38: 77-84.

28 Huang CT, Ho CC, Tsai YJ, et al. Factors influencing visibility and diagnostic yield of transbronchial biopsy using endobronchial ultrasound in peripheral pulmonary lesions. Respirology 2009; 14: 859-864.

29 Yoshikawa M, Sukoh N, Yamazaki K, et al. Diagnostic value of endobronchial ultrasonography with a guide sheath for peripheral pulmonary lesions without X-ray fluoroscopy. Chest 2007; 131: 1788-1793.

30 Yamada N, Yamazaki K, Kurimoto N, et al. Factors related to diagnostic yield of transbronchial biopsy using endobronchial ultrasonography with a guide sheath in small peripheral pulmonary lesions. Chest 2007; 132: 603-608.

31 Bilaceroglu S, Kumcuoglu Z, Alper $\mathrm{H}$, et al. CT bronchus sign-guided bronchoscopic multiple diagnostic procedures in carcinomatous solitary pulmonary nodules and masses. Respiration 1998; 65: 49-55.
32 Priola AM, Priola SM, Cataldi A, et al. Accuracy of CT-guided transthoracic needle biopsy of lung lesions: factors affecting diagnostic yield. La Radiologia Medica 2007; 112: 1142-1159.

33 Li H, Boiselle PM, Shepard JO, et al. Diagnostic accuracy and safety of CT-guided percutaneous needle aspiration biopsy of the lung: comparison of small and large pulmonary nodules. AJR Am J Roentgenol 1996; 167: 105-109.

34 Kothary N, Lock L, Sze DY, et al. Computed tomography-guided percutaneous needle biopsy of pulmonary nodules: impact of nodule size on diagnostic accuracy. Clin Lung Cancer 2009; 10: 360-363.

35 Tsukada H, Satou T, Iwashima A, et al. Diagnostic accuracy of CTguided automated needle biopsy of lung nodules. AJR Am J Roentgenol 2000; 175: 239-243.

36 Hiraki T, Mimura H, Gobara H, et al. CT fluoroscopy-guided biopsy of 1,000 pulmonary lesions performed with 20-gauge coaxial cutting needles: diagnostic yield and risk factors for diagnostic failure. Chest 2009; 136: 1612-1617.

37 Yeow KM, Tsay PK, Cheung YC, et al. Factors affecting diagnostic accuracy of CT-guided coaxial cutting needle lung biopsy: retrospective analysis of 631 procedures. J Vasc Interv Radiol 2003; 14: 581-588.

38 Arslan S, Yilmaz A, Bayramgurler B, et al. CT-guided transthoracic fine needle aspiration of pulmonary lesions: accuracy and complications in 294 patients. Med Sci Monit 2002; 8: CR493-CR497.

39 Cox JE, Chiles C, McManus CM, et al. Transthoracic needle aspiration biopsy: variables that affect risk of pneumothorax. Radiology 1999; 212: 165-168.

40 Kazerooni EA, Lim FT, Mikhail A, et al. Risk of pneumothorax in CT-guided transthoracic needle aspiration biopsy of the lung. Radiology 1996; 198: 371-375.

41 Heyer CM, Reichelt S, Peters SA, et al. Computed tomographynavigated transthoracic core biopsy of pulmonary lesions: which factors affect diagnostic yield and complication rates? Acad Radiol 2008; 15: 1017-1026.

42 Yeow KM, Su IH, Pan KT, et al. Risk factors of pneumothorax and bleeding: multivariate analysis of $660 \mathrm{CT}$-guided coaxial cutting needle lung biopsies. Chest 2004; 126: 748-754.

43 Hiraki T, Mimura H, Gobara $\mathrm{H}$, et al. Incidence of and risk factors for pneumothorax and chest tube placement after CT fluoroscopyguided percutaneous lung biopsy: retrospective analysis of the procedures conducted over a 9-year period. AJR Am J Roentgenol, 194: 809-814.

44 Yildirim E, Kirbas I, Harman A, et al. CT-guided cutting needle lung biopsy using modified coaxial technique: factors effecting risk of complications. Eur J Radiol 2009; 70: 57-60.

45 Laurent F, Michel P, Latrabe V, et al. Pneumothoraces and chest tube placement after CT-guided transthoracic lung biopsy using a coaxial technique: incidence and risk factors. AJR Am J Roentgenol 1999; 172: 1049-1053.

46 Topal U, Ediz B. Transthoracic needle biopsy: factors effecting risk of pneumothorax. Eur J Radiol 2003; 48: 263-267.

47 Khan MF, Straub R, Moghaddam SR, et al. Variables affecting the risk of pneumothorax and intrapulmonal hemorrhage in CTguided transthoracic biopsy. Eur Radiol 2008; 18: 1356-1363.

48 Fukushima A, Ashizawa K, Aso N, et al. [CT-guided needle biopsy of the lung: factors affecting risk of complications]. Nihon Igaku Hoshasen Gakkai zasshi 2001; 61: 96-99.

49 Ko JP, Shepard JO, Drucker EA, et al. Factors influencing pneumothorax rate at lung biopsy: are dwell time and angle of pleural puncture contributing factors? Radiology 2001; 218: 491-496. 